\title{
Educación Física y promoción de la salud: estrategias de intervención en la escuela Physical Education and healthpromotion: strategies of intervention in the school
}

\author{
Lourdes Rué Rosell, Maria Ángeles Serrano Alfonso \\ Universitat de Barcelona
}

\begin{abstract}
Resumen: Este trabajo pretende profundizar, desde una perspectiva multidisciplinar, en las posibilidades de estrategias e intervenciones que ofrece la Educación Física en el contexto educativo, concretamente en la educación primaria, y que contribuyen al establecimiento de hábitos saludables en el alumnado y que pueden extenderse más allá del propio alumnado. La identificación de tales intervenciones se ha realizado a partir de una revisión profunda de las bases de datos que recogen las publicaciones de más impacto internacional a través de la Web of Knowledge. Asimismo, se presenta la potencial aplicabilidad de estas prácticas, por compartir algunas de las características que las definen o por ser propuestas ya compartidas, en centros educativos que se han transformado en Comunidades de Aprendizaje.
\end{abstract}

Palabras clave: Educación Física, Educación Primaria, Estrategias de intervención en la escuela, Promoción de la salud, Comunidades de aprendizaje

Abstract: This paper focus, from a multidisciplinary approach, on the strategies and interventions that physical education offers in the establishment of healthy habits in the educative context, specifically in primary education. Physical education is contributing to establish healthy habits among students and can be extended further the students, to the community. The identification of these strategies and interventions has been done through the literature review of publications with the highest international impact and which are collected on the ISI Web of Knowledge. Moreover, the paper also presents the applicability of these interventions to educative centers that have decided to transform into Learning Communities, because sharing some of their characteristics or proposals.

Key words: Physical Education, Primary Education, Strategies of intervention in the school, Health promotion, Learning Communities.

\section{Introducción}

Desde la educación, las posibilidades que nos brinda la Educación Física son extraordinariamente amplias. A lo largo de las últimas décadas, la Educación Física se ha considerado una área de gran importancia e interés en el desarrollo de valores y actitudes que implícitamente se trabajan en esta materia. Es también innegable, su importancia para el desarrollo del bienestar y la salud del alumnado. Pero a su vez, la Educación Física ofrece un enorme potencial en el desarrollo de prácticas saludables y hábitos para la promoción de la salud.

Este artículo profundiza en el estudio de qué prácticas educativas existen en la educación primaria, que se sirvan de la Educación Física, cuál es su propósito y qué factores inciden y se contemplan en estas intervenciones. Se ha examinado principalmente quépapel tiene la Educación Física y qué aportaciones pueden derivarse de ello para el fomento de una sociedad del futuro y del presente que goce de más salud, haciendo hincapié en la necesidad de contemplar aquellos grupos más vulnerables.

El trabajo que presentamos se estructura en cinco apartados. En primer lugar, se presentan los objetivos del mismo y el contexto actual de la investigación en esta área; en segundo lugar, la metodología utilizada que corresponde a una revisión de la literatura científica internacional basándose en las publicaciones recogidas a la Web of Knowledge; posteriormente, se presentan los resultados de la mencionada revisión de la literatura científica; que a continuación, se discuten; y finalmente, se exponen las conclusiones.

\section{Objetivos}

Los objetivos de este artículo son, por un lado, profundizar en el estudio de las estrategias y acciones que se sirven de la Educación Física en contextos educativos, y por otro lado, analizar el potencial de estas prácticas y su incidencia en la mejora de la salud del alumnado y la comunidad. Asimismo también se relacionan estas actuaciones en el marco del proyecto educativo de Comunidades de Aprendizaje que, por sus características, es un contexto óptimo y de gran interés.

Fecha recepción: 05-12-13- Fecha envío revisores: 05-12-13- Fecha de aceptación: 10-12-13 Marta Capllonch Bujosa

Campus Mundet, Edifici Llevant, 3a planta, despatx 317,

Passeig del Vall d'Hebron 171, 08035 Barcelona,

mcapllonch@ub.edu

\section{Contexto actual de la investigación}

La Educación Física en la escuela se considera el medio más eficaz e inclusor para que todos los niños y niñas puedan adquirir y aprender las habilidades, el conocimiento y los valores de su participación a lo largo de sus vidas, con independencia de su capacidad, sexo, edad, cultura, raza/etnia, religión o nivel social (ICSSPE, 2010).

Concebimos laEducación Física no como simplemente una materia que se imparte en el contexto escolar sino como una disciplina cuyas prácticas realizadas en este marco pueden ir más allá del aula y del alumnado, pudiendo ampliar su campo de actuación al resto de la comunidad educativa. Es por este motivo, y por los rasgos característicos que definen las Comunidades de Aprendizaje, que el presente trabajo expone como estos centros de educación primaria pueden ser un contexto adecuado para ampliar y extender la aplicabilidad potencial que nos ofrece la Educación Física en la promoción de la salud.

Comunidades de Aprendizaje responde a un proyecto basado en actuaciones educativas de éxito dirigidas a la transformación social y educativa. Se definen como:

(...) un proyecto de transformación social y cultural de un centro educativo y de su entorno, para conseguir una sociedad de la información para todas las personas, basada en el aprendizaje dialógico, mediante la educación participativa de la comunidad que se concreta en todos sus espacios incluida el aula (Valls, 2000, p.8).

Las Comunidades de Aprendizaje implican a todas las personas que directa o indirectamente influyen en el aprendizaje y desarrollo de los estudiantes, se incluye así a profesorado, familiares, amistades, vecinos del barrio, miembros de asociaciones, etc. Este modelo educativo, cuenta actualmente, con más de 120 centros educativos que son Comunidades deAprendizaje, habiéndose extendido a nivel internacional, llegando por ejemplo hasta Brasil. Asimismo, han sido objeto de estudio en el marco del proyecto de investigación INCLUD-ED. Strategies for inclusion and social cohesion from education in Europe, del Sexto Programa Marco de Investigación de la Unión Europea, como una actuación de éxito para el fomento de la cohesión social en Europa a través de la educación (CREA, 2006-2011). La transformación de los centros educativos en Comunidades de Aprendizaje converge con el doble objetivo de superar el fracaso escolar y mejorar la convivencia. Debido a ello, se trata de centros educativos con unas particularidades que hacen todavía más necesario profundizar en la potencialidad que ofrece la Educación Física en el contexto educativo, ya que muchos de 
estos centros se encuentran en zonas socio-económicamente deprimidas y/ o cuentan con un elevado índice de inmigración. Además, el propio proyecto que estos centros asumen por ser Comunidades de Aprendizaje se caracteriza por abrirse a la participación de toda la comunidad y requiere la necesidad y el compromiso de transformar su entorno para que sea un espacio generador de posibilidades. Es decir, las Comunidades de Aprendizaje buscan todos los recursos y posibilidades que se encuentran en la comunidad para acrecentar los aprendizajes de todo el alumnado (Díez-Palomar \& Flecha, 2010). De manera que examinar qué actuaciones se realizan desde la Educación Física en el contexto educativo es todavía más fundamental en entornos más desfavorecidos, como pueden ser aquellos en los que encontramos centros educativos que son Comunidades de Aprendizaje.

La creciente diversidad del alumnado en las escuelas de educación primaria, conlleva el reto educativo de que adquieran los conocimientos y habilidades necesarias para hacer frente a las necesidades actuales y futuras de los estudiantes. Uno de los aspectos más importantes hace referencia a la adquisición y promoción de actitudes saludables, donde la actividad y la Educación Física resultan fundamentales. Generar o promover hábitos saludables en edades tempranas es un factor clave para mejorar el estado de salud del alumnado pero también para poder asentar la base de que este alumnado en su etapa de vida adulta continúe desarrollando una vida saludable. En el presente artículo abordaremos cómo la Educación Física puede incidir en la promoción de la salud desde diferentes ópticas, y cómo esta cuestión está deviniendo un asunto de índole internacional y una preocupación desde la salud pública.

Los beneficios de la actividad física son incuestionables y bien fundamentados, gozando de consenso científico internacional. La participación regular en estas actividades se asocia a una mayor, más prolongada y mejor calidad de vida, a una reducción de los riesgos de padecer una serie de enfermedades, así como a un bienestar emocional y psicológico (WHO / ICSSPE, 2004). La práctica regular de deporte y actividades físicas también incide en el desarrollo educativo e intelectual y en la inclusión social (WHO / ICSSPE, 2004).

En 1998, la World Health Organization (WHO) en la Fifty-first World Health Assembly, enunciaba que la salud es uno de los derechos fundamentales de los seres humanos. Gozar de salud y de bienestar depende de la interrelación de muchos factores, entre los que el estilo de vida es el más importante (Religioni \& Czerw, 2012). En este sentido, encontramos diversas iniciativas que persiguen promover la salud de las personas desde la prevención y educación para la salud. Es el caso de Healthy People, donde el plan 2020, contempla la necesidad de considerar aspectos como la educación y la pobreza, entre otros factores sociales que influyen en la salud de las personas y en conseguir la equidad en salud (Koh, Piotrowski, Kumanyika \& Fielding, 2011).

A pesar de los beneficios innegables que supone la práctica de la actividad física, actualmente existe también una gran preocupación por la inactividad física de niños y jóvenes, y de la población en general. Se trata de una preocupación creciente a nivel internacional y que se traduce, por ejemplo, en el aumento de niños y jóvenes con sobrepeso y/o obesidad o con riesgo de ello, y de todas las consecuencias que de esta situación se derivan ya que la inactividad física es también uno de los factores de riesgo de determinadas enfermedades.

Se sabe ya que la inactividad física es el cuarto factor de riesgo de mortalidad más importante en todo el mundo. La inactividad física aumenta en muchos países, y ello influye considerablemente en la prevalencia de enfermedades no transmisibles (ENT) y en la salud general de la población mundial (Organización Mundial de la Salud, 2010).

La Asamblea Mundial de la Salud, en 2004, apoyó la resolución WHA57.17: Estrategia Mundial sobre Régimen Alimentario, Actividad Física y Salud (WHA57.17, 2004), recomendando el desarrollo de planes de acción y políticas nacionales por parte de los Estados Miembros para aumentar la práctica de actividad física de sus poblaciones. La importancia de la actividad física para la salud pública evidencia la necesidad de definir y aplicar directrices nacionales y políticas que promuevan el ejercicio físico y la prevención de las ENT.

Desde Europa, se ha diseñado el Plan de Trabajo Europeo para el Deporte para 2011-2014. En este plan se presentan tres ejes prioritarios que deberán poner en marcha los Estados miembros y la Comisión, uno de estos hace referencia a los valores sociales del deporte donde se contempla aumentar la actividad física y la práctica deportiva para el fomento de la salud. El plan está conectado con el Libro Blanco sobre el Deporte que es la primera iniciativa a nivel europeo sobre el deporte como fenómeno social y económico.

Desde el panorama español, también hay esfuerzos en la misma dirección. El Plan A+D, Plan Integral para la Actividad Física y el Deporte (Ministerio de Educación, Cultura y Deporte - Consejo Superior de Deportes, 2010), se ha elaborado considerando los criterios y orientaciones de organismos internacionales de relevancia sobre la temática, de manera que las Directrices de Actividad Física de la Unión Europea y las Recomendaciones de la OMS sobre «Actividad Física y Salud» son las principales referencias internacionales del PlanA+D. El mencionado Plan es un instrumento creado con la voluntad de garantizar el acceso universal a la práctica de la actividad física y del deporte a la totalidad de la población española, para ayudar a frenar el alto índice de sedentarismo y obesidad e impulsar hábitos de vida más saludables y activos. El Plan A+D, fruto también de la participación y colaboración con distintos organismos de carácter público y privado, presenta una serie de líneas de actuación para la década 2010-2020. También presenta algunos datos reveladores. Es el caso de las cifras del Eurobarómetro 2009 que muestran que en España la práctica de la actividad física y deportiva se encuentra por debajo de la media europea y con un elevado índice de sedentarismo; así como los datos de la OMS que también indican que España es el país europeo con una mayor prevalencia de obesidad y sobrepeso infantil (26.3\% en las edades entre 2 y 24 años; y 35\% para niños y niñas de 13 años).

En la misma línea, los datos del Instituto Nacional de Estadística (INE) revelan la progresión de la obesidad y el sobrepeso de la población española. La Encuesta Nacional de Salud 2011-2012(ENSE)(INE, 2013) saca a la luz que el $41.3 \%$ de la población adulta española se declara sedentaria, subrayando una vez más que es uno de los países con más alto nivel de sedentarismo de Europa, y como la obesidad ha aumentado del 7.4\% al 17\% en los últimos 25 años. En el caso de la población adulta, se constata el aumento de sobrepeso y obesidad (tanto en el caso de hombres como de mujeres) donde el 53.7\% de la población española adulta padece obesidad o sobrepeso (63.15\% en hombres y 44.18\% en mujeres); en la población infantil, de 2 a 17 años, se observa como el $27.8 \%$ de esta población padece obesidad o sobrepeso, es decir, de cada 10 niños y adolescentes de 2 a 17 años, dos tienen sobrepeso y uno obesidad(INE, 2013). La Encuesta Europea de Salud en España. Año 2009 (EES09), a diferencia de la anterior no recoge datos de la población infantil, sólo mayores de 16 años, también refleja que más de la mitad de las personas adultas supera su peso recomendado (INE, 2010).

El aumento del sobrepeso y/o la obesidad están siendo una realidad preocupante y compartida a nivel internacional. La WHO estima que 1.9 millones de muertes en todo el mundo son debidas a la inactividad física. También destaca que enfermedades crónicas como el cáncer, la diabetes y las enfermedades cardíacas coronarias están relacionadas con la inactividad física(WHO, 2002). En la misma línea, a escala mundial, se constata que la obesidad infantil y la inactividad física están aumentando. Se ha estimado que en el 2015 uno de cada cinco niños tendrá sobrepeso (Janssen, Toussaint, Van Willem \& Verhagen, 2011).

Frente a este panorama, la inactividad física y la obesidad infantil representan un grave problema de salud, una preocupación desde la salud pública a nivel internacional. Así que, urge empezar a abordar esta problemática, y para hacerlo, la escuela es el espacio más idóneo. Aunque la práctica de la Educación Física no siempre es la deseable, la escuela puede desarrollar una labor fundamental en aumentar los niveles de la práctica de la actividad física y del deporte en los escolares. 


\section{Material y métodos}

Para llevar a cabo la revisión de la literatura científica internacional sobre los programas de salud en el contexto educativo, especialmente en la educación primaria, y vinculados a la Educación Física se ha realizado una búsqueda a través de las bases de datos que recogen las publicaciones de más impacto internacional a través de la Web of Knowledge. Concretamente, se han cruzado en la categoría de topic, y acotando el intervalo de tiempo en los últimos cinco años, los siguientes términos: health programme AND primary education AND physical education, obteniendo 650 resultados que han sido analizados atendiendo a los objetivos del presente trabajo y que a continuación se presentan.

\section{Resultados}

Presentamos en este apartado los resultados de la búsqueda realizada. Son varias las líneas de investigación centradas en el ámbito de la Educación Física y de la salud en el contexto educativo.Acontinuación, más allá de las que estudian un tipo determinado de enfermedad o se centran en sus pacientes se presentan aquellas que de modo general son de interés para nuestro estudio y que se dirigen a la globalidad de nuestra población de interés (la totalidad del alumnado y la comunidad educativa).

Antes de empezar a presentar los resultados, consideramos de interés señalar la presencia de estudios que se sirven de las nuevas tecnologías para la promoción de la salud. Constituyen una línea de investigación aparecida de forma reciente, pero que con toda seguridad se desarrollará mucho más próximamente. En la interacción educaciónpromoción de la salud, algunas investigaciones están contemplando las aportaciones que desde las nuevas tecnologías, como el uso de Internet y otras formas de tecnologías, están contribuyendo a la promoción de la salud, por ejemplo en la población adolescente (Tercyak, Abraham, Graham, Wilson \& Walker, 2009). Estudios recientes, como la investigación de Cavallo, Tate, Ries, Brown, DeVellis \& Ammerman(2012) se focaliza en la eficacia de las redes sociales como factor que puede ayudar a incrementar la actividad física, mientras otros como el de Fukuoka et al. (2011) parten de las posibilidades que ofrece la telefonía móvil para promover intervenciones relacionadas con la práctica de la actividad física.

En el contexto educativo, la gran mayoría de los estudios se centran en la educación primaria y secundaria, constatamos así esfuerzos en la línea de prevención y para afrontar la obesidad y/o sobrepeso en los niños de edad escolar y adolescentes. Como hemos mencionado, también el presente trabajo se centra en la educación primaria. No obstante, creemos que es de interés destacar que existen estudios y programas, aunque no son muy abundantes, sobre la importancia de la actividad física en el alumnado preescolar (Niederer, et al., 2009; Roth, et al., 2010; Jones, et al., 2011; Nethe, et al., 2012). Los beneficios de una actividad física regular son incuestionables. Loprinzi, Cardinal, Loprinzi \& Lee (2012) observan que una participación regular en la actividad física durante la infancia tiene no únicamente numerosos beneficios inmediatos sobre la salud, sino que también tiene efectos duraderos en la edad adulta. En otras palabras, sugieren que los beneficios de la actividad física en la infancia también parecen influir positivamente en los resultados de salud de los adultos.

¿Qué actuaciones y/o programas vinculados a la Educación Física y la salud se están realizando en el ámbito de la educación primaria?

Existe una diversidad de programas y actuaciones asociadas a la Educación Física que se están llevando a cabo en el contexto educativo. Hemos identificado multitud de intervenciones y programas escolares centrados en temáticas específicas que se ocupan de dicha cuestión desde diferentes ámbitos de actuación. En este sentido, una de las líneas de investigación más consolidadas se centra en la prevención del sobrepeso u obesidad del alumnado a través de la actividad física; una segunda línea de actuación se dirige a la evaluación y eficacia de los programas e intervenciones escolares que han sido implementados; otras intervenciones escolares amplían sus destinatarios a la comunidad educativa; otras también tienen en cuenta la incidencia del entorno en que se producen; $\mathrm{y}$ finalmente otros estudios se focalizan en los grupos más vulnerables.

\section{Física}

Intervenciones y programas escolares centrados en la Educación

Desde una perspectiva preventiva

Existen múltiples intervenciones escolares en clave de prevención y para reducir la obesidad o favorecer la pérdida de peso, con el fin de tener un peso corporal saludable mediante la promoción de la actividad física. Moreno \& Gracia-Marco (2012) señalan la actividad física como factor de prevención para la obesidad infantil. También en la misma línea, existen estudios que profundizan sobre los efectos de la Educación Física en niños de edad escolar y en cómo aumentar sus niveles de actividad física para mejorar su salud (Janssen, et al., 2011; Sigmund, El Ansari \& Sigmundová, 2012). Con posterioridad a la realización de una revisión sistemática y análisis de los programas de intervención en obesidad infantil y los componentes de éxito de estos programas, AlMarzooqi \& Nagy (2011) aseveraron que las escuelas son los mejores escenarios para realizar intervenciones de obesidad infantil. Los mismos autores, afirman que estas actuaciones deberían centrarse en mejorar la actividad física y promover la nutrición saludable, para que su efecto se refleje en una mejor salud y bienestar de los niños.

Los resultados obtenidos del trabajo de Molinero, Castro-Pinero, Ruiz, González Montesinos, Mora \& Márquez (2010), confirman la necesidad de una adecuada educación en los hábitos de estilo de vida y de desarrollar programas de intervención en niños y jóvenes con relación a la dieta y la actividad física, poniendo el acento en que se dirijan especialmente a chicas ya que son menos activas físicamente. Partiendo de esta realidad, el trabajo de Okely et al. (2011) se focaliza también en intervenciones escolares para promover y sostener la actividad física entre las chicas adolescentes ya que en ellas los niveles de actividad física disminuyen considerablemente.

Considerando la actividad física en la línea de medicina preventiva encontramos el estudio de Telford et al. (2009). En el marco del cual investigaron los estilos de vida de niños y jóvenes y cómo la actividad física contribuye a su salud y desarrollo. También sobre estilos de vida saludables con relación a la actividad física, el consumo de frutas y verduras, la constitución corporal, el conocimiento y variables psicológicas, hallamos la investigación de Gorely, Nevill, Morris, Stensel \& Nevill (2009). Los autores también señalan que las escuelas son un lugar adecuado para la promoción de estilos de vida saludables.

\section{Desde una perspectiva de la evaluación y eficacia}

Otra de las líneas de interés identificadas en la revisión de la literatura, se basa en examinar y evaluar de forma rigurosa las intervenciones que se están llevando a cabo. Así, encontramos distintos programas que son evaluados. En Chile, Kain, Leyton, Cerda, Vio y Uauy (2009) evaluaron la eficacia de un programa de prevención de obesidad escolar, cuya intervención abarcaba tanto actividades relacionadas con la nutrición como con la actividad física en niños chilenos de educación primaria. También con el propósito de examinar la efectividad de intervenciones escolares preventivas de la obesidad, el trabajo de Verstraeten et al. (2012) realiza una revisión sistemática sobre el comportamiento alimentario y/o la actividad física para una prevención primaria de la obesidad en niños y adolescentes. Los resultados de su trabajo indican que las intervenciones escolares tienen la capacidad de mejorar las conductas alimentarias y la actividad física.

Otros estudios centrados en la mejora y evaluación de intervenciones y programas en relación con la actividad física sobre la salud en el alumnado de primaria son el estudio de Eather, Morgan y Lubans (2011) y el de Moores (2010). Ambos contemplan la evaluación para demostrar que existen iniciativas efectivas para la promoción de la salud en el contexto educativo.

Desde la óptica de evaluación, el trabajo de Watson-Jarvis, Johnston y Clark (2011) versa sobre la evaluación de un programa para familias 
con niños en riesgo de sobrepeso y obesidad. Sus resultados sugirieron que fue una intervención efectiva.

Desde un punto de vista más escéptico, encontramos el trabajo de Baker, Francis, Soares, Weightman y Foster (2011) que evalúa los efectos de intervenciones comunitarias, sugiriendo que los efectos de algunas intervenciones con relación a los niveles poblacionales de la actividad física son inconsistentes, por lo que no existen evidencias de que tales intervenciones aumenten eficazmente los niveles de actividad física de la población. El estudio de De Meij et al. (2010), parte de la falta de estrategias de intervenciones escolares eficaces que promuevan la actividad física. Uniendo investigación sobre las intervenciones y su ejecución real, es decir, llevarlas a la práctica, pretenden arrojar luz sobre los efectos o no de las intervenciones.

Una revisión sistemática de la efectividad de intervenciones escolares fue realizada por Brown y Summerbell (2009). Su estudio centrado en la alimentación y los niveles de actividad física para prevenir la obesidad infantil concluye que las intervenciones en los niveles de actividad física en la escuela pueden ser de ayuda para mantener un peso saludable aunque los resultados son inconsistentes y a corto plazo. No obstante, señalan también que las intervenciones escolares en que se dé la combinación de actividad física y dieta saludable pueden ser preventivas frente al sobrepeso a largo plazo.

\section{Desde una perspectiva de la comunidad educativa}

Encontramos desde esta perspectiva, trabajos que se dirigen no únicamente al alumnado de primaria, si no que consideran también otros miembros de la comunidad educativa como el profesorado de escuelas de educación primaria. La influencia de este colectivo se puede observar, desde una nueva aproximación, teniendo en cuenta el desarrollo de los programas curriculares de Educación Física y cómo estos pueden incidir en el desarrollo saludable del alumnado (Thorburn, Carse, Jess \& Atencio, 2011). También partiendo del colectivo del profesorado, el estudio australiano de Rosenkranz et al. (2012) ha profundizado en las estrategias que pueden favorecer la práctica de la actividad física en los adolescentes. Los autores constatan que hay poco conocimiento sobre las estrategias del profesorado que efectivamente motiven al alumnado a participar en la actividad física, aunque la influencia del profesorado es clave.

Otros programas incluyen, además del profesorado, a otros miembros de la comunidad educativa como las madres y padres. Es el caso del programa escolar «Komm mit in das gesunde Boot - Grundschule», diseñado frente a la necesidad de actuar preventivamente y de forma temprana en aumentar la actividad física y reducir el tiempo dedicado al sedentarismo impulsando alternativas saludables, y para potenciar un estilo de vida saludable(Dreyhaupt, et al., 2012). La necesidad de incluir a las madres y padres en las prácticas de mejora de la actividad física de los niños es de gran relevancia y contribuye al éxito de las intervenciones ejecutadas en la obesidad infantil (Ball, et al., 2012). En la misma línea, destacan programas escolares que han sido eficaces, pero cuya eficacia ha sido mejorada gracias a la inclusión de las madres y los padres (Nyberg, Sundblom, Norman \& Elinder, 2011).

La intervención My Parenting SOS fue diseñada, realizada y evaluada por Ward et al. (2011). Se trata de un estudio centrado en la familia como medio de prevención o de cambio frente a la obesidad infantil. Profundiza en la influencia de los padres hacia sus hijas/os con respecto a los hábitos saludables.

El objetivo del estudio de Morgan et al. (2011) era evaluar la viabilidad y eficacia del programa 'Healthy Dads, Healthy Kids' (HDHK), cuyo diseño pretendía ayudar a los padres con sobrepeso a perder peso y a ser un modelo de conducta saludable positivo para sus hijos. Los autores valoraron positivamente el programa HDHK ya que se mejoró la salud de los padres y la actividad física de los niños.

La intervención CHANGE! (Children's Health, Activity, and Nutrition: Get Educated!) se centra en la fase formativa de hábitos saludables y los destinatarios del mismo son tanto personas adultas (madres, padres y profesorado) como alumnado de las escuelas del programa CHANGE! En el diseño de este programa de intervención debemos destacar el estudio de Mackintosh, Knowles, Ridgers y Fairclough (2011) que tuvo en cuenta la información recogida a partir de la participación de los distintos centros educativos de primaria sobre actividad física. Los resultados demuestran que existe una comprensión de la relación entre actividad física y salud, así como la percepción del tiempo invertido por la familia que fue positivamente vinculado a la participación en actividad física. Se concluye que las familias tienen un rol importante en promover entornos saludables. De manera que la participación de los padres, y de toda la familia, es una estrategia que podría ser significativa para aumentar los niveles de actividad física de los niños.

Eichhorn et al. (2012) presentan la iniciativa llamada «BEO’S», así como su implementación y aceptación en las escuelas participantes. BEO's contempla, de forma similar a los casos anteriores, el conjunto de la comunidad educativa, su participación y se adapta a los recursos de los que dispone el centro educativo y a sus necesidades. La intención del programa es la de mejorar los ambientes de la escuela así como favorecer conductas alimentarias más saludables y aumentar la actividad física de los estudiantes con el fin de prevenir la obesidad y mejorar su salud.

McCarron et al. (2010), constataron como muchos de los programas dirigidos a combatir el sobrepeso entre los jóvenes americanos eran elaborados por «expertos» mientras que la perspectiva de las familias, y comunidades, que son las más afectadas y con más posibilidad de cambiar las conductas eran raramente contempladas. Así establecieron Shaping America’s Youth (SAY) donde se abordó la nutrición y la actividad física dando cabida a la participación de la familia y la comunidad.

\section{Desde una perspectiva del contexto}

Otras intervenciones van más allá de la escuela estrictamente y de la comunidad educativa, y se ocupan del entorno como un factor incidente en la promoción de la actividad física y que puede acompañar a la escuela en su labor de prevención de la obesidad infantil y promoción de la actividad física. Así lo indican De Bourdeaudhuij et al. (2011) que señalan como la combinación de componentes educativos y ambientales dan efectos más relevantes para la promoción de la actividad física.

También desde un sentido amplio del entorno escolar, la investigación de De Villiers et al.(2012) evalúa el entorno de la escuela de primaria con relación a si se dan buenas prácticas nutritivas, suficiente Educación Física, prevención del hábito de fumar. Los autores apuntan que existen factores clave del contexto que pueden tener un rol importante en los comportamientos saludables del alumnado, los padres y los educadores. Por lo que se señala la influencia del contexto escolar en materia de salud.

Aarts, van de Goor, van Oers y Schuit (2009) apuestan por la creación de vecindades «activas» para la promoción de la salud de los padres y niños. Cradock, Kawachi, Colditz, Gortmaker y Buka (2009) profundizan también en el papel de los barrios y cómo estos pueden tener un rol destacado en apoyar conductas saludables a la población. Los autores concluyeron que la cohesión social del vecindario influencia en la participación de la actividad física.

Desde una perspectiva de centrarse en comunidades vulnerables

Existe una relación entre el nivel socio-económico y la calidad de vida y la salud de las personas. Esta realidad, se traduce en que un bajo estatus socioeconómico va asociado a valores más bajos en medidas referentes a la calidad de vida y la salud. Por este motivo, distintas investigaciones se centran en comunidades o grupos más vulnerables que son más susceptibles de padecer estas situaciones.

Pappa, Kontodimopoulos, Papadopoulos y Niakas (2009) evaluaron la influencia de factores demográficos y socio-económicos sobre la salud y la calidad de vida en el contexto griego. Sus resultados evidencian la relación existente entre estos factores.

En la investigación de Mier et al. (2013), centrada en un grupo de niños mejico-americanos, se constata que este grupo de niños se encuentra mucho más afectado por la obesidad en comparación con otros grupos poblacionales. También después de observar cómo había pro- 
gresado la prevalencia de sobrepeso en adolescentes africo-americanos, la iniciativa Challenge! nacía con fines preventivos y era administrada por college mentors (Black, et al. 2012), un recurso de gran valor y de éxito para la intervención.

El estudio de Pitrou, Shojaei, Wazana, Gilbert y Kovess-Masfety (2010) se basa en la prevalencia de sobrepeso infantil con relación a una muestra de niños en edad escolar de educación primaria en Francia. Los autores sugieren que los programas de prevención escolares contra el sobrepeso deberían de ser implementados primero en las áreas desaventajadas.

\section{Discusión}

La escuela es el espacio más idóneo e igualitario para trabajar desde la Educación Física, la promoción de la salud y de hábitos de vida saludables, así como la consecución de valores y favorecer la inclusión de todo el alumnado. Todo ello es especialmente relevante cuando nos referimos a alumnado perteneciente a grupos vulnerables.

La revisión de la literatura científica evidencia la estrecha relación entre el nivel socioeconómico y educativo de la población con las medidas referentes a la salud y la calidad de vida. Asimismo, actualmente se constata también que la inactividad física está aumentando en muchos países manifestándose en el estado de salud de la población mundial. En las últimas décadas y en los países desarrollados, la prevalencia de la obesidad y/o el sobrepeso en niños y jóvenes ha aumentado de forma alarmante. Prevalencia que es mayor en el caso de niños y jóvenes pertenecientes a grupos poblaciones de colectivos más vulnerables.

Frente a la urgencia de poner freno a los índices crecientes del sobrepeso y/o obesidad infantil, y al panorama que se perfila de ello en la sociedad en general, distintos estudios profundizan en intervenciones escolares que de la mano de la Educación Física en la escuela ahondan en esta cuestión y ofrecen alternativas saludables. Estos estudios indican que la Educación Física es una medida efectiva para la prevención y/o tener un peso corporal saludable, así como en clave de futuro. Con respecto a la eficacia y evaluación de estas prácticas escolares se constata la necesidad de que deben ser evaluadas para seguir impulsando aquellas que ofrezcan mejores resultados. Fruto también de la revisión de la literatura científica, hemos constatado como la participación de toda la comunidad educativa, profesorado, padres, madres, entre otros, e incluso el entorno en que se circunscribe el centro educativo o el contexto escolar en general, inciden en la práctica y en el éxito de las intervenciones realizadas que contemplan la Educación Física. En la misma línea, de incluir toda la comunidad educativa, encontramos los centros escolares que son Comunidades de Aprendizaje. Las Comunidades deAprendizaje cuentan con la implicación de toda la comunidad educativa y representan espacios de potencial aplicabilidad de estas prácticas. Desde estos centros educativos existe ya una sensibilización y preocupación por estas cuestiones que hasta la fecha se han reflejado especialmente en la alimentación. Sin embargo, rasgos característicos de las Comunidades deAprendizaje como son la consideración de toda la comunidad educativa y de sus necesidades, que se trata de un proyecto de transformación social y educativo por lo que traspasa las fronteras del centro e incide en el entorno, y que se tratan de centros educativos que muchas veces se encuentran en zonas deprimidas y con un alumnado diverso y perteneciente a grupos desfavorecidos, hacen aún más necesario profundizar en acciones que promuevan la Educación Física para la mejora de la salud de su alumnado y de toda la comunidad en general.

\section{Conclusiones}

La literatura científica está explorando las posibilidades que ofrece la Educación Física desde el marco educativo. Todas ellas coinciden en la importancia de la actividad física para la salud pública. En este sentido, apuestan por promover la práctica del ejercicio físico, y los hábitos de vida saludables en general, como medida de prevención y de estilo de vida saludables para hacer frente al aumento del sobrepeso y/o la obesidad, así como a las enfermedades relacionadas con ello.
Esta es una cuestión de actualidad a nivel internacional pero también es una cuestión de futuro, ya que el sobrepeso y/o la obesidad infantil y la inactividad física están aumentando alarmantemente. Frente a este panorama, la comunidad científica está realizando investigaciones centradas en sus respectivos objetivos de interés y a partir de las que pretende hallar respuestas.

La revisión de la literatura científica realizada nos revela que la escuela es el espacio más apropiado para trabajar y fomentar hábitos saludables a todo el alumnado, donde el aumento de la práctica de la actividad física y el deporte escolar son esenciales. Las investigaciones de hacen eco de ello, y en consecuencia existen estudios enmarcados en los distintos niveles educativos.

Educar en la adquisición de hábitos saludables contempla mejorar la educación para la promoción de estilos de vida saludables, así como la práctica y la Educación Física en la escuela. Para ello, se deben desarrollar eimplementar las intervenciones escolares que sean más apropiadas al contexto y que den respuesta a las necesidades de la comunidad educativa, urge especialmente la implementación de estas prácticas por ejemplo en el caso de grupos más desfavorecidos o en zonas socioeconómicamente deprimidas. Se ha constatado también que las prácticas escolares que incluyen la participación de toda la comunidad educativa son más eficaces y contribuyen al éxito de las mismas, aquí es especialmente relevante el papel de la familia. En este sentido, las Comunidades de Aprendizaje son un contexto óptimo y de interés para seguir profundizando en la potencialidad de la implementación de estas intervenciones, ya que por sus propias características los beneficios de dichas actividades se podrían reflejar también en toda la comunidad educativa y el entorno. Bajo esta perspectiva, pretendemos seguir investigando.

La promoción de la educación y la actividad física en la escuela, no incide únicamente en un buen desarrollo de los niños y adolescentes sino que también supone asentar las bases para que en su vida adulta gocen de una mejor salud y calidad de vida.

\section{Referencias:}

Aarts, M.J., Van de Goor, I.A.M., Van Oers, H.A.M., \& Schuit,A.J.(2009). Towards translation of environmental determinants of physical activity in children into multi-sector policy measures: study design of a Dutch project. BMC Public Health, 9. doi: 10.1186/1471-2458-9-396

AlMarzooqi, M.A., \& Nagy, M.C. (2011). Childhood Obesity Intervention Programs: A Systematic Review. Life Science Journal - Acta Zhengzhou University Overseas Edition, 8(4), 45-60.

Baker, P.R.A., Francis, D.P., Soares, J., Weightman, A.L., \& Foster, C. (2011). Community wide interventions for increasing physical activity. Cochrane Database of Systematic Reviews, 4. doi: 10.1002/14651858.CD008366.pub2

Ball, GD.C., Ambler, K.A., Keaschuk, R.A., Rosychuk, R.J., Holt, N.L., . . \& Newton, A S. (2012). Parents as Agents of Change (PAC) in pediatric weight management: The protocol for the PAC randomized clinical trial. BMC Pediatrics, 12. doi: 10.1186/ 1471-2431-12-114

Black, M.M., Arteaga, S.S., Sanders, J., Hager, E.R.,Anliker, J.A. Gittelsohn, J., \& Wang, Y. (2012). College mentors: a view from the inside of an intervention to promote health behaviors and prevent obesity among low-income, urban, African American adolescents. Health promotion practice, 13(2), 238-44. doi: 10.1177/ 1524839910385899

Brown, T., \& Summerbell, C. (2009). Systematic review of school-based interventions that focus on changing dietary intake and physical activity levels to prevent childhood obesity: an update to the obesity guidance produced by the National Institute for Health and Clinical Excellence. Obesity Reviews, 10(1), 110-141. doi: 10.1111/ j.1467-789X.2008.00515.X

Cavallo, D.N. Tate, D.F, Ries, A. V Brown, J.D DeVellis, R.F, \& Ammerman, A.S. (2012) A Social Media-Based Physical Activity Intervention A Randomized Controlled Trial. American Journal of Preventive Medicine, 43(5), 527-532. doi: 10.1016/ j.amepre.2012.07.019

Cradock, A.L., Kawachi, I., Colditz, G.A., Gortmaker, S.L., \& Buka, S.L. (2009) Neighborhood social cohesion and youth participation in physical activity in Chicago. Social Science \& Medicine, 68(3), 427-435. doi: 10.1016/ j.socscimed.2008.10.028

De Bourdeaudhuij, I., Van Cauwenberghe, E., Spittaels, H., Oppert, J.M., Rostami, C.,... \& Maes, L. (2011). School-based interventions promoting both physical activity and healthy eating in Europe: a systematic review within the HOPE project. Obesity Reviews, 12(3), 205-216. doi: 10.1111/j.1467-789X.2009.00711.x

De Meij, J.S.B., Chinapaw, M.J.M., Kremers, S.P.J., Van der Wal, M.F., Jurg, M.E., \& Van Mechelen, W. (2010). Promoting physical activity in children: the stepwise development of the primary school-based JUMP-in intervention applying the REAIM evaluation framework. British Journal of Sports Medicine, 44(12), 879-887. 
doi: 10.1136/bjsm.2008.053827

De Villiers, A., Steyn, N.P., Draper, C.E., Fourie, J.M., Barkhuizen, G., . . \& Lambert, E.V. (2012). «HealthKick»: Formative assessment of the health environment in lowresource primary schools in the Western Cape Province of South Africa. BMCPublic Health, 12. doi: 10.1186/1471-2458-12-794

Díez-Palomar, J., \& Flecha, R. (2010). Comunidades de Aprendizaje: un proyecto de transformación social y educativa. Revista Interuniversitaria de Formación del Profesorado, 67(24,1), 19-30.

Dreyhaupt, J., Koch, B., Wirt, T., Schreiber, A., Brandstetter, S.,. . . \& Steinacker, J.M. (2012) Evaluation of a health promotion program in children: Study protocol and design of the cluster-randomized Baden-Wurttemberg primary school study [DRKS-ID: DRKS00000494]. BMC Public Health, 12. doi: 10.1186/1471-2458-12-157

Eather, N., Morgan, P.J., \& Lubans, D.R. (2011). Improving health-related fitness in children: the fit-4-Fun randomized controlled trial study protocol. BMC Public Health, 11. doi: 10.1186/1471-2458-11-902

Eichhorn, C., Bodner, L., Liebl, S., Scholz, U., Wozniak, D., .. \& Loss, J. (2012). BEO’S - Physical Activity and Healthy Eating at Schools in Oberfranken, Bavaria Concept and First Results of a Resource-Oriented, Systemic Approach in School-Based Health Promotion. Gesudheitswesen, 74(2), 104-111. doi: 10.1055/s-0031 1275708

Encuesta Europea de Salud en España. Año 2009. Instituto Nacional de Estadística (INE) / Ministerio de Sanidad, Política Social e Igualdad, 2010. Recuperado de: http://www.ine.es/jaxi/menu.do?type=pcaxis\&path=\%2Ft15/p420\&file=inebase

Encuesta Nacional de Salud 2011-2012 (ENSE). Instituto Nacional de Estadística (INE) / Ministerio de Sanidad, Servicios Sociales e Igualdad, 2013.

Fukuoka, Y., Komatsu, J., Suarez, L., Vittinghoff, E., Haskell, W., Noorishad, T., \& Pham, K. (2011). The mPED randomized controlled clinical trial: applying mobile persuasive technologies to increase physical activity in sedentary women protocol. BMC Public Health, 11. doi: 10.1186/1471-2458-11-933

Gorely, T., Nevill, M.E., Morris, J.G, Stensel, D.J., \& Nevill, A. (2009). Effect of a schoolbased intervention to promote healthy lifestyles in 7-11 year old children. International Journal of Behavioral Nutrition and Physical Activity, 6. doi: 10.1186/1479-5868-6-5

INCLUD-ED. Strategies for inclusion and social cohesion from education in Europe Integrated Project Priority 7 of Sixth Framework Programme, European Commission (2006-2011).

International Position Statement on Physical Education (2010, noviembre). International Council of Sport Science and Physical Education (ICSSPE). Recuperado de:

http://www.icsspe.org/content/international-position-statement-physical-education

Janssen, M., Toussaint, H.M., Van Willem, M., \& Verhagen, E.A.L.M. (2011). PLAYgrounds: Effect of a PE playground program in primary schools on PA levels during reces in 6 to 12 year old children. Design of a prospective controlled trial. BMC Public Health, 11. doi: 10.1186/1471-2458-11-282

Jones, R.A., Riethmuller, A., Hesketh, K., Trezise, J., Batterham, M., \& Okely, A.D. (2011) Promoting Fundamental Movement Skill Development and Physical Activity in Early Childhood Settings: A Cluster Randomized Controlled Trial. Pediatric Exercise Science, 23(4), 600-615.

Kain, J., Leyton, B., Cerda, R, Vio, F., \& Uauy, R. (2009). Two-year controlled effectiveness trial of a school-based intervention to prevent obesity in Chilean children. Public Health Nutrition, 12(9), 1451-1461. doi: 10.1017/S136898000800428X

Koh, H.K.; Piotrowski, J.J.; Kumanyika, S., \& Fielding, J. (2011). Healthy People: A 2020 Vision for the Social Determinants Approach. Health Education \& Behavior, 38, 6, 551-557. doi: 10.1177/1090198111428646

Loprinzi, P.D., Cardinal, B.J., Loprinzi, K.L., \& Lee, H. (2012). Benefits and Environmental Determinants of Physical Activity in Children and Adolescents. Obesity Facts, 5(4), 597-610. doi: 10.1159/000342684

Mackintosh, K.A., Knowles, Z.R., Ridgers, N.D., \& Fairclough, S.J. (2011).Using formative research to develop CHANGE!: a curriculum-based physical activity promoting intervention. BMC Public Health, 11. doi: 10.1186/1471-2458-11-831

McCarron, D.A., Richartz, N., Brigham, S., White, M.K., Klein, S.P., \& Kessel, S.S. (2010). Community-Based Priorities for Improving Nutrition and Physical Activity in Childhood. Pediatrics, 126, 73-89. doi: 10.1542/peds.2010-0482C

Mier, N., Smith, M.L., Irizarry, D., Carrillo-Zuniga, G., Lee, C., Trevino, L., \& Ory, M.C. (2013). Bridging Research and Policy to Address Childhood Obesity Among Border Hispanics A Pilot Study. American Journal of Preventive Medicine, 44(3), S208-S214. doi: 10.1016/j.amepre.2012.11.013

Molinero, O., Castro-Pinero, J., Ruiz, J.R., González Montesinos, J.L., Mora, J., \& Márquez, S. (2010). Health Behaviour of School Children at the Province of Cadiz. Nutrición Hospitalaria, 25(2), 280-289. doi: 10.3305/nh.2010.25.2.4579

Moores, P.S. (2010). Engaging Community Partners to Promote Healthy Behaviours in Young Children. Canadian Journal of Public Health-Revue Canadienne de Sante Publique, 101(5), 369-373

Moreno, L.A., \& Gracia-Marco, L. (2012). Prevención de la obesidad desde la actividad física: del discurso teórico a la práctica [Obesity prevention from physical activity: from theoretical discourse to practice]. Anales de pediatria (Barcelona, Spain: 2003), 77, 2, 136.e1-6. doi: 10.1016/j.anpedi.2012.04.011

Morgan, P.J., Lubans, D.R., Callister, R., Okely, A.D., Burrows, T.L., Fletcher, R., \& Collins, C.E. (2011). The 'Healthy Dads, Healthy Kids' randomized controlled trial: efficacy of a healthy lifestyle program for overweight fathers and their children. International Journal of Obesity, 35(3), 436-447. doi: 10.1038/ijo.2010.151

Nethe, A., Dorgelo, A., Kugelberg, S., Van Assche, J., Buijs, G.,... \& Manios, Y. (2012) Existing policies, regulation, legislation and ongoing health promotion activities related to physical activity and nutrition in pre-primary education settings: an overview. Obesity Reviews, 13, 118-128. doi: 10.1111/j.1467-789X.2011.00963.x

Niederer, I. Kriemler, S., Zahner, L., Bürrgi,F.,Ebenegger, V.,. . \& \& Puder,J.J.(2009). Influence of a lifestyle intervention in preschool children on physiological and psychological parameters (Ballabeina): study design of a cluster randomized controlled trial. BMC Public Health, 9. doi: 10.1186/1471-2458-9-94

Nyberg, G, Sundblom, E., Norman, A., \& Elinder, L.S. (2011). A healthy school startParental support to promote healthy dietary habits and physical activity in children: Design and evaluation of a cluster-randomised intervention. BMC Public Health, 11. doi: $10.1186 / 1471-2458-11-185$

Okely, A.D., Cotton, W.G., Lubans, D.R., Morgan, P.J., Puglisi, L., .. \& \& Perry, J. (2011). A school-based intervention to promote physical activity among adolescent girls: Rationale, design, and baseline data from the Girls in Sport group randomised controlled trial. BMC Public Health, 11. doi: 10.1186/1471-2458-11-658

Organización Mundial de la Salud (OMS). (2010). Recomendaciones mundiales sobre actividad física para la salud.

Pappa, E., Kontodimopoulos, N., Papadopoulos, A.A., \& Niakas, D. (2009). Assessing the socio-economic and demographic impact on health-related quality of life: evidence from Greece. International Journal of Public Health, 54(4), 241-249. doi: 10.1007/s00038-009-8057-x

Pitrou, I., Shojaei, T., Wazana, A., Gilbert, F., \& Kovess-Masfety, V. (2010). Child Overweight, Associated Psychopathology, and Social Functioning: A French School-based Survey in 6-to 11-year-old Children. Obesity, 18(4), 809-817. doi: 10.1038/oby.2009.278

Plan Integral para la Actividad Física y el Deporte. Ministerio de Educación, Cultura y Deporte - Consejo Superior de Deportes, 2010. Recuperado de:

http://www.csd.gob.es/csd/sociedad/plan-integral-para-la-actividad-fisica-y-el-deporte-plan-a-d/

Religioni, U., \& Czerw, A. (2012). Health promotion in the context of National Health Programme to 2015. Progress in Health Sciences, 2(2), 167-173.

Resolución WHA57.17. Estrategia Mundial sobre Régimen Alimentario, Actividad Física y Salud. En: $57^{a}$ Asamblea Mundial de la Salud, Ginebra, 17 a 22 de mayo de 2004. Resoluciones y decisiones, anexos. Ginebra, OMS, 2004.

Rosenkranz, R.R. Lubans, D.R., Peralta, L. R., Bennie, A., Sanders, T, \& Lonsdale, C. (2012). A cluster-randomized controlled trial of strategies to increase adolescents' physical activity and motivation during physical education lessons: the Motivating Active Learning in Physical Education (MALP) trial. BMC Public Health, 12. doi: 10.1186/1471-2458-12-834

Roth, K., Mauer, S., Obinger, M., Ruf, K.C., Graf, C., .. . \& Hebestreit, H. (2010). Prevention through Activity in Kindergarten Trial (PAKT): A cluster randomised controlled trial to assess the effects of an activity intervention in preschool children. BMC Public Health, 10, doi: 10.1186/1471-2458-10-410

Sigmund, E., El Ansari, W., \& Sigmundová, D. (2012). Does school-based physical activity decrease overweight and obesity in children aged 6-9 years? A two-year non-randomized longitudinal intervention study in the Czech Republic. BMC Public Health, 12. doi: 10.1186/1471-2458-12-570

Telford, R.D., Bass, S.L., Budge, M. M., Byrne, D. G., Carlson, J.S., .. \& \& Waring, P. (2009). The lifestyle of our kids (LOOK) project: outline of methods. Journal of Science and Medicine in Sport, 12(1), 156-163. doi: 10.1016/j.jsams.2007.03.009

Tercyak, K.P., Abraham, A.A., Graham, A.L., Wilson, L.D., \& Walker, L.R. (2009) Association of Multiple Behavioral Risk Factors with Adolescents Willingness to Engage in eHealth Promotion. Journal of Pediatric Psychology, 34(5), 457469. doi: $10.1093 /$ jpepsy/jsn085

Thorburn, M., Carse, N., Jess, M., \& Atencio, M. (2011). Translating change into improved practice: Analysis of teachers' attempts to generate a new emerging pedagogy in Scotland. European Physical Education Review, 17(3), 313-324. doi: 10.1177/ 1356336X11416727

Valls, R. (2000). Comunidades de Aprendizaje. Una práctica educativa de aprendizaje dialógico para la sociedad de la información. Tesis Doctoral. Universitat de Barcelona.

Verstraeten, R., Roberfroid, D., Lachat, C., Leroy, J.L., Holdsworth, M., Maes, L., \& Kolsteren, P.W. (2012). Effectiveness of preventive school-based obesity interventions in low- and middle-income countries: a systematic review. American Journal of Clinical Nutrition, 96(2), 415-438. doi: 10.3945/ajcn.112.035378

Ward, D.S., Vaughn, A.E., Bangdiwala, K.I., Campbell, M., Jones, D.J., Panter, A.T., \& Stevens, J. (2011). Integrating a family-focused approach into child obesity prevention: Rationale and design for the My Parenting SOS study randomized control trial. BMC Public Health, 11. doi: 10.1186/1471-2458-11-431

Watson-Jarvis, K., Johnston, C., \& Clark, C. (2011). Evaluation of a Family Education Program For Overweight Children and Adolescents. Canadian Journal of Dietetic Practice and Research, 72(4), 191-196. doi: 10.3148/72.4.2011.191

World Health Organization (WHO) / International Council of Sport Science and Physica Education (ICSSPE). (2004). Girls' Participation in Physical Activities and Sports: Benefits, Patterns, Influences and Ways Forward. Canterbury Christ Church University College, UK: Bailey, R., Wellard, I., \& Dismore, H. Recuperado de: http://www.icsspe.org/content/who-benefits-physical-activity

World Health Organization (WHO). (2002). The world health report 2002 - Reducing Risks, Promoting Healthy Life. Recuperado de:

http://www.who.int/whr/2002/en/whr02_en.pdf

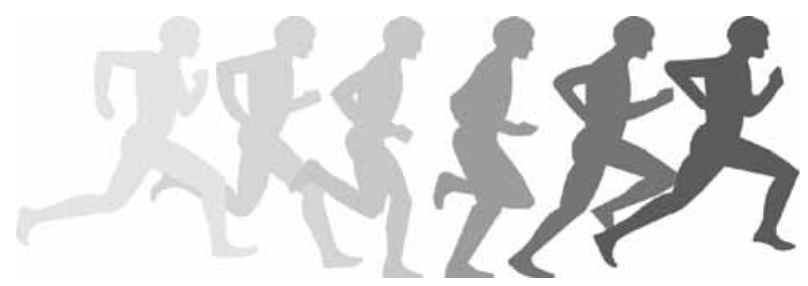

